\title{
Análise de Modelos de Balanced Scorecard elaborados a partir da Ótica da Sustentabilidade através do uso da Matriz SWOT ${ }^{1}$
}

\author{
Analysis of Balanced Scorecard Models elaborated from \\ Sustainability perspective through SWOT Diagram
}

\author{
Maria Daniella de Oliveira Pereira da Silva \\ Professora Assistente da Universidade Federal da Paraíba, \\ Centro de Tecnologia e Desenvolvimento Regional \\ Endereço: Centro de Tecnologia e Desenvolvimento (UFPB) \\ Regional, Cidade Universitária - Campus I, Bessa \\ CEP: 58.051-900 - João Pessoa, PB - Brasil \\ Telefone: (83) 3216-7927 \\ e-mail: mariadaniella75@hotmail.com
}

\author{
Antônio André Cunha Callado \\ Professor Associado II do Departamento de Administração \\ da Universidade Federal Rural de Pernambuco \\ Endereço: Universidade Federal Rural de Pernambuco \\ Departamento de Administração. \\ Rua, Dom Manuel de Medeiros, S/N, Dois Irmãos, CEP: \\ 52.171-030, Recife, PE - Brasil, Telefone: (81) 33206447 \\ e-mail: andrecallado@yahoo.com.br
}

\section{RESUMO}

Este artigo teve o objetivo de analisar modelos de Balanced Scorecard elaborados a partir da ótica da sustentabilidade através do uso da Matriz SWOT. Para a operacionalização desta pesquisa foi realizada uma pesquisa bibliográfica para identificar modelos de Balanced Scorecard específicos voltados para aspectos relacionados à sustentabilidade empresarial. Foram encontrados vinte e um artigos científicos que faziam a apresentação de modelos de Balanced Scorecard voltados para a questão ambiental, dos quais apenas três artigos se enquadraram no critério de elegibilidade estabelecido. Posteriormente, foi operacionalizada a análise SWOT referente à adaptabilidade dos modelos encontrados para empresas agroindustriais. A partir dos resultados obtidos, foi possível visualizar as diferenças existentes entre os três modelos encontrados.

Palavras-chave: Balanced Scorecard. Balanced Scorecard Sustentável. Análise SWOT.

\begin{abstract}
This article aimed to analyze Balanced Scorecard models developed from the perspective of sustainability through the use of SWOT diagram. The operationalization of this research was carried out by literature search to identify Balanced Scorecard models focused on specific aspects related to corporate sustainability. Twenty-one papers that presented Balanced Scorecard models focused on environmental issues were found, of which only three items fitted in the eligibility criteria given. SWOT analysis regarding the adaptability of the models found for agribusiness companies was performed. The results obtained show that it was possible to visualize the differences between the three models found.
\end{abstract}

Keywords: Balanced Scorecard. Sustainable Balanced Scorecard. SWOT analysis.

\footnotetext{
1 Artigo recebido em 23.10.2013. Revisado pelos pares em 12.11.2013 (blind review). Aceito para publicação em 20.11.2013. Recomendado para publicação por José Ribamar Marques de Carvalho (Editor Científico). Publicado em 12.12.2013. Organização responsável UACC/CCJS/UFCG.
} 
SILVA, M. D. de O. P.; CALlADO, A. A. C. Análise de Modelos de Balanced...

\section{INTRODUÇÃO}

Com o processo de globalização e o aumento da competitividade empresarial, as empresas passaram a ter a necessidade de controlar informações relacionadas aos seus aspectos financeiros e aos seus aspectos não financeiros, como a responsabilidade social e ambiental da empresa.

A integração de aspectos sustentáveis a metodologias de avaliação de desempenho estratégico passaram a ser realizadas por diversos pesquisadores, exemplificando o caso das incorporações das questões sustentáveis ao Balanced Scorecard, possibilitando, com isso, não só o controle dinâmico e a coordenação das áreas de responsabilidade da empresa, como também, possibilitando uma visão do futuro da empresa (BARNEGAS et al, 2000).

A avaliação do desempenho socioambiental pode ser usada para estimar o desempenho competitivo empresarial (JOHNSON, 1998). O Balanced Scorecard, nesse sentido, pode ser usado, não só para auxiliar o processo de mensuração da gestão estratégica de sustentabilidade empresarial, como também, poderá possibilitar que incipiências relacionadas às informações de caráter gerencial sejam supridas (MACEDO; QUEIROZ, 2007). Ainda, o Balanced Scorecard pode auxiliar esse processo de mensuração, por possibilitar a integração das questões ambientais junto à estratégia da empresa (CAMPO; SELIG, 2002).

Entretanto, o Balanced Scorecard deve ser visto como uma metodologia flexível, que deve ter as suas métricas alteradas sempre que a estratégia sustentável modificar (JOHNSON, 1998). Assim, as métricas do Balanced Scorecard com visão sustentável deverão ser adaptadas de acordo com as necessidades empresariais.

De acordo com Hockerts e O'Rourke (2002) as razões para a existência do Balanced Scorecard com ênfase sustentável estão relacionadas as possibilidade dessa metodologia:

- Medir e controlar os impactos sociais diretos e indiretos;

- Gerir o relacionamento empresarial com diferentes grupos interessados;

- Compreender o impacto da concorrência com as questões sociais, através do seu sistema de transformação como o mercado, a pressão das políticas públicas, as indústrias e as atividades voluntárias.

- Analisar e prever o ciclo de vida das questões sociais e as suas consequências para o campo competitivo.

Já segundo a visão de Quesado e Rodrigues (2009), umas das principais razões para o surgimento dessas metodologias é a possibilidade de estabelecer elementos de medição qualitativa, para estabelecer indicadores de desempenho ambiental, identificando os diferentes responsáveis pelo processo de gestão ambiental. Rocha, Neves e Selig (2001, p. 2) reforçam esta perspectiva ao afirmarem que indicadores de 
desempenho ambiental podem possibilitar assim, “[...] um eficiente gerenciamento ambiental".

Nesse sentido, Macedo e Queiroz (2007) afirmam que o Balanced Scorecard é um instrumento abrangente que possibilita a utilização de estratégias sustentáveis. Por ser um sistema aberto, o Balanced Scorecard permite a incorporações de questões sustentáveis através dos ajustes nas suas perspectivas, (MOLLER; SCHALTEGGER, 2005). O Balanced Scorecard sustentável surge no sentido de complementar à visão do Balanced Scorecard tradicional, possibilitando que a empresa possa fazer a união das questões relacionadas à sustentabilidade junto à estratégia global da empresa.

De maneira similar ao Balanced Scorecard tradicional proposto por Kaplan e Norton (1997), o Balanced Scorecard sustentável deve ser entendido como uma ferramenta gerencial que pode ser aplicada em qualquer setor de atividade econômica. A partir desta vertente, o objetivo deste trabalho foi analisar modelos de Balanced Scorecard elaborados a partir da ótica da sustentabilidade através do uso da Matriz SWOT.

\section{REFERÊNCIAL TEÓRICO}

\subsection{Balanced Scorecard}

O Balanced Scorecard segundo Kaplan e Norton (1997, p. 19) é definido como "um instrumento que integra medidas financeiras derivadas das estratégias, sem menosprezar as medidas financeiras do desempenho passado [...]". Enquanto, Barbosa Junior (2005, p. 39) define o Balanced Scorecard como sendo "Indicadores Balanceados de Desempenho [...] que não deve se limitar a informações econômicas e financeiras".

Já segundo Martinsons, Davison e Tse (1999, p. 72), “o próprio nome do Balanced Scorecard reflete o seu conceito, o qual tem a intenção de manter em equilíbrio, um conjunto de itens". O que também pode ser explicado como um equilíbrio entre medidas financeiras relacionadas ao desempenho passado da empresa, equilibradas junto às medidas dos fatores que impulsionam o desempenho futuro (GIONGO, 2004).

O BSC corresponde a uma ferramenta gerencial voltada para mensuração do desempenho empresarial, através da organização de medidas financeiras e medidas não financeiras, as quais são alinhadas estrategicamente através de perspectivas, para a obtenção do objetivo empresarial a curto, médio e longo prazos.

Pelo fato do Balanced Scorecard utilizar conceitos já existentes, ou seja, fazer uma organização de elementos empresariais pré-existentes, a ferramenta sofre diversas críticas, como Costa (2001, p. 135) que afirma que o Balanced Scorecard “[...] 
não traz qualquer conceito novo, nunca antes tratado na literatura contábil, mas repensa temas antigos, chamando atenção para pontos vitais que não estavam claramente articulados".

Partindo dessa perspectiva que o Balanced Scorecard não é algo novo, Giuntini (2003, p.98) afirma que, o mesmo, corresponde a "[...] uma evolução de teorias já existentes, como: Tableau de Board, Critério de Excelência do Prêmio Malcolm Baldrige, Reengenharia e Qualidade Total." A ferramenta organiza elementos das teorias no sentido de formar uma Teoria de Gestão, através da união de elementos táticos, operacionais e estratégicos alinhados à visão e a missão da empresa.

Dessa forma, o Balanced Scorecard organiza os objetivos monetários e não monetários a uma sequência de ações, envolvendo o financeiro, os processos internos e os clientes junto ao aspecto organizacional, de forma a proporcionar a longo prazo, o desempenho econômico esperado (KAPLAN; NORTON, 1997).

O sucesso de um Balanced Scorecard está associado não só a combinação de medidas financeiras e medidas não financeiras nas perspectivas (GREBIM, 2004). Como também a associação dessas medidas junto às perspectivas, com o enfoque nos objetivos estratégicos de longo prazo da empresa (SOARES, 2001). Assim, o Balanced Scorecard "[...] deve ser visto como a instrumentação de uma estratégia única" (KAPLAN; NORTON, 1997, p.169).

Os objetivos e as medidas de cada uma das perspectivas sozinhas não atingem o objetivo do Balanced Scorecard, que corresponde à comunicação da estratégia empresarial para todos os níveis da organização. As perspectivas devem ser integradas, pois ao contrário poderão otimizar apenas o foco da perspectiva individual, contribuindo parcialmente para o sucesso do desempenho empresarial (BARBOSA JUNIOR, 2006).

O Balanced Scorecard possibilita a medição do desempenho empresarial (SILVA, 2006), através das perspectivas, as quais são estruturadas com objetivos, medidas ou indicadores, metas e iniciativas (HERRERO, 2005). Entretanto, o Balanced Scorecard não corresponde a um sistema de controle, mas a "[...] um sistema de comunicação, informação e aprendizado [...]", (KAPLAN; NORTON, 1997, p. 25). Dessa forma, não existe um plano preestabelecido para controlar as entidades, cada uma deve estruturar o seu Balanced Scorecard de acordo com a sua visão e com a sua estratégia empresarial.

Portanto, no Balanced Scorecard o gerenciamento da estratégia é um processo contínuo, no qual os gestores precisam monitorar os objetivos, as medidas, as metas e as iniciativas de cada perspectiva (MARIAN et al, 2008). A fim de avaliar se é preciso realizar alguma atualização ou substituição, de acordo com a visão atualizada da estratégia (KAPLAN; NORTON, 1997). Assim, como verificar se os objetivos propostos estão sendo alcançados (CARVALHO; TOSTES, 2007). 
Uma vez que as mudanças relacionadas ao mercado e a estrutura interna da entidade, podem desencadear mudanças na estratégia empresarial. Dessa forma, é fundamental a realização de um constante feedback nas iniciativas estratégicas das perspectivas do Balanced Scorecard. Deste forma, para as empresas que adotam o Balanced Scorecard, é fundamental que sejam realizadas reavaliações das metas voltadas para os clientes, processos internos, financeiras, funcionários e sistemas.

\subsection{Balanced Scorecard Sustentável}

A percepção do Balanced Scorecard sob o aspecto sustentável foi apresentada inicialmente por Azzone et al. (1996) e Johnson (1998). Posteriormente, Figge et al. (2002), Bieker e Waxenberger (2002), Bieker e Gminder (2002), Sidiropoulos et al. (2004), Durán e Pluglia (2007) e Länsiluoto e Järvenpää (2007) também o abordaram como ferramenta gerencial ambiental.

A literatura apresenta abordagens diferenciadas quanto às terminologias sugeridas para o Balanced Scorecard com a ênfase na sustentabilidade, tais como Banced Scorecard Ambiental (JOHNSON, 1998; DURÁN; PLUGLIA, 2007; QUESADO; RODRIGUES, 2009), Balanced Scorecard Verde (LÄNSILUOTO; JÄRVENPÄÄ, 2007), Eco Balanced Scorecard (SIDIROPOULOS et al., 2004), Eco eficiente Balanced Scorecard (HOCKERS: 2001) e Balanced Scorecard Sustentável (BIEKER; WAXENBERGER, 2002; BIEKER; GMINDER, 2002).

A incorporação de aspectos da gestão ambiental no Balanced Scorecard é algo de interesse para muitas empresas, pois possibilita a inclusão de indicadores não financeiros para a organização (QUESADO; RODRIGUES, 2009). Além disso, possibilita fomentar o diálogo na gestão da organização sobre os aspectos estratégicos direcionando-os a um foco (ZINGALES; HOCKERTS, 2003), desencadeado, consequentemente, diversas vantagens para a organização.

Entretanto, não existe uma única nomenclatura para os modelos propostos baseados no Balanced Scorecard associado à gestão ambiental, assim como não existe um modelo específico. O que mostra que ainda não existe um consenso entre os pesquisadores quanto aos aspectos terminológicos e quanto à formação do modelo, mesmo existindo "uma popularidade sobre o conceito do Balanced Scorecard Sustentável entre grupos de pesquisadores e industriais europeus", como afirma Hamner (2005, p. 13).

Assim, os modelos de Balanced Scorecard com ênfase na sustentabilidade podem ser estruturados através de quatro possibilidades, segundo a visão dos autores Figge et al (2002), Monteiro, Castro e Prochnik (2003) e Sidiropoulos et al (2004), que são: 
- $1^{\text {a }}$ Possibilidade - Modelos com a inclusão de indicadores ambientais na perspectiva dos processos internos ou mudanças nas perspectivas tradicionais, através da incorporação de indicadores voltados para aspectos sustentáveis.

- 2 ${ }^{\text {a }}$ Possibilidade: Modelos que propõem a inclusão de indicadores ambientais nas perspectivas tradicionais do Balanced Scorecard.

- $3^{\text {a }}$ Possibilidade: Modelos que propõem a criação perspectivas complementares às perspectivas tradicionais, com ênfase na sustentabilidade e na gestão ambiental.

- 4 a a Possibilidade: Modelos voltados para a criação de um Balanced Scorecard específico para departamentos, como por exemplo, a criação de modelos de Balanced Scorecard para os departamentos de gestão ambiental das organizações.

Deste modo, pode-se assumir que não existe uma única possibilidade de estruturar o Balanced Scorecard para mensurar a gestão estratégica associada aos aspectos sustentáveis e que, com isso, existam diversas propostas de modelos, que podem diferenciar-se um dos outros.

\section{PROCEDIMENTOS METODOLÓGICOS}

A presente pesquisa, quanto aos meios de realização, foi classificada como uma pesquisa bibliográfica por realizar uma investigação sobre modelos de Balanced Scorecard Sustentável. Segundo Vergara (2009) e Gil (2009) esse tipo de estudo toma como base materiais publicados em livros, revistas, redes eletrônicas, entre outros meios. Para a realização do estudo foram pesquisados materiais associados a modelos de Balanced Scorecard específicos voltados para atender a sustentabilidade empresarial.

Segundo Andrade (2003), as fontes bibliográficas são formadas por diversos tipos de documentos, como os documentos manuscritos, impressos, documentos que reproduzem outros documentos (gravações de áudio e vídeo) e documentos cartográficos ou fotográficos.

Gil (2009) classifica essas fontes bibliográficas como: livros de leitura corrente (livros literários, científicos e técnicos); obras de referência (enciclopédia, dicionários, manuais); periódicos científicos; teses e dissertações; anais de encontros científicos; periódicos de indexação e resumo.

Assim, as fontes utilizadas na elaboração da pesquisa foram livros científicos, periódicos nacionais e internacionais, teses, dissertações e anais de encontros científicos. 
A localização das fontes bibliográficas pode ser dada através de: bibliotecas convencionais, bases de dados em formato eletrônico e sistema de busca eletrônica (GIL, 2009).

Dessa forma, para a realização do estudo foram realizadas as coleta de material em bibliotecas (livros e periódicos), em bases de dados e em sistemas de busca. As bases de dados eletrônicos usadas foram o Scientific Eletronic Library Online - SciELO e o portal de periódicos da Coordenação de Aperfeiçoamento de Pessoal de Nívlel Superior - CAPES. Na coleta de material foi utilizado também o sistema de busca eletrônica Google Acadêmico. Contudo, para a realização da pesquisa não foi levado em consideração nenhum periódico específico, uma vez que o assunto referente ao Balanced Scorecard Sustentável é escasso.

Na busca de materiais, realizadas nas bases eletrônicas, foram considerados os seguintes termos na língua portuguesa, inglesa e espanhola: "Balanced Scorecard Ambiental", "Eviromental Balanced Scorecard", "Sustentability Balanced Scorecard", "Balanced Scorecard Sustentável", "Cuadro de mandos integrados", "EBSC", "BSCA", "BSCS" e "SBSC". Os artigos referentes ao Balanced Scorecard sustentável foram classificados nas seguintes categorias:

- Trabalhos voltados para a inclusão de indicadores ambientais apenas na perspectiva dos processos internos ou mudanças nas perspectivas tradicionais;

- Trabalhos voltados para a inclusão de indicadores ambientais nas perspectivas tradicionais do Balanced Scorecard;

- Trabalhos voltados para a criação perspectivas complementares as perspectivas tradicionais, com ênfase na gestão ambiental;

- Trabalhos voltados para a criação de um Balanced Scorecard específico.

Os artigos que apresentam modelos de Balanced Scorecard elaborados especificamente para a abordagem sustentável foram analisados a partir da matriz SWOT em suas quatro perspectivas, a saber:

- Forças;

- Fraquezas;

- Oportunidades;

- Ameaças.

\section{APRESENTAÇÃO E DISCUSSÃO DOS RESULTADOS}

Primeiramente, foi realizada a uma busca eletrônica por referências bibliográficas que tratassem de Balanced Scorecard sustentável. Os resultados desta pesquisa estão apresentados no quadro 1. 
SILVA, M. D. de O. P.; CALlADO, A. A. C. Análise de Modelos de Balanced...

\begin{tabular}{|c|c|}
\hline Categorias & Referências encontradas \\
\hline $\begin{array}{l}\text { Modelos com a inclusão de indicadores ambientais apenas } \\
\text { na perspectiva dos processos internos ou mudanças nas } \\
\text { perspectivas tradicionais }\end{array}$ & $\begin{array}{l}\text { - Johnson (1998) } \\
\text { - Hockerts (2001) } \\
\text { - Länsiluoto e Järvenpää (2007). }\end{array}$ \\
\hline $\begin{array}{l}\text { Modelos com a Inclusão de indicadores ambientais no } \\
\text { modelo tradicional do Balanced Scorecard }\end{array}$ & $\begin{array}{l}\text {-Barnegas et al (2000) } \\
\text { - Hockerts e O'Rouke (2002) } \\
\text { - Duran e pluglia (2008) } \\
\text { - Laurinkevièiute et al (2008) }\end{array}$ \\
\hline $\begin{array}{l}\text { Modelos com a criação de perspectivas complementares } \\
\text { ao modelo tradicional do Balanced Scorecard }\end{array}$ & $\begin{array}{l}\text { - Bieker e Gminder (2001) } \\
\text { - Bieker, Dyllick, Gminder \& } \\
\text { hockerts (2001) } \\
\text { - Figge et al. (2002) } \\
\text { - Bieker (2002) } \\
\text { - Beja (2003) } \\
\text { - Sidiroupolos et al (2004) } \\
\text { - Möller e Schaltegger (2005) } \\
\text { - Bieker (2005) } \\
\text { - García (2007) } \\
\text { - Hubbard (2009) } \\
\text { - Macedo e Queiroz (2007) }\end{array}$ \\
\hline $\begin{array}{l}\text { Modelos com a criação de um Balanced Scorecard específico } \\
\text { para mensurar o desempenho da gestão empresarial } \\
\text { sustentável }\end{array}$ & $\begin{array}{l}\text { - Campos e Selig (2002) } \\
\text {-Yongvanich e Guthrie (2006) } \\
\text { - Bonacchi e Rinaldo (2007) }\end{array}$ \\
\hline
\end{tabular}

Quadro 1 - Resultado da pesquisa bibliográfica

Pode-se observar que foram encontradas 21 referências, dentre as quais 3 apresentam a criação de modelos de Balanced Scorecard sustentável: Bonacchi e Rinaldo (2007); Yongvanich e Guthrie (2006); e Campos e Selig (2002).

Os modelos foram avaliados quanto a sua aplicabilidade através de uma análise de SWOT, na qual foram levantadas as principais forças e fraquezas dos modelos, e também as oportunidades e ameaças dos mesmos. Caracterizando assim, o ambiente interno e externo, assim como os aspectos positivos e negativos dos modelos.

Inicialmente, foi analisado o modelo de Balanced Scorecard sustentável proposto por Campos e Selig (2002). Os resultados estão apresentados no quadro 2. 
SILVA, M. D. de O. P.; CALlADO, A. A. C. Análise de Modelos de Balanced...

\begin{tabular}{|c|c|c|}
\hline \multirow[b]{2}{*}{ 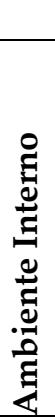 } & \multicolumn{2}{|c|}{ Negativo } \\
\hline & $\begin{array}{l}\text { FORÇAS } \\
\text { - Uso de teorias ligadas aos } \\
\text { aspectos ambientais. } \\
\text { - Estrutura voltada para mais de } \\
\text { um tipo de atividade. } \\
\text { - Apresentação da mensuração do } \\
\text { modelo. }\end{array}$ & $\begin{array}{l}\text { FRAQUEZAS } \\
\text { - Estrutura complexa. } \\
\text { - Necessidade de muito } \\
\text { capital humano. } \\
\text { - Desvalorização dos } \\
\text { aspectos sociais. } \\
\text { - Ênfase na parte operacional }\end{array}$ \\
\hline 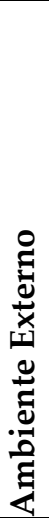 & $\begin{array}{l}\text { OPORTUNIDADES } \\
\text { - Possibilidade de obtenção de } \\
\text { certificações ambientais. } \\
\text { - Expansão para mercados } \\
\text { futuros. } \\
\text { - Obtenção da sustentabilidade } \\
\text { empresarial com a valorização dos } \\
\text { aspectos sociais. } \\
\text { - Expansão da sustentabilidade } \\
\text { entre as empresas. }\end{array}$ & $\begin{array}{l}\text { AMEAÇAS } \\
\text { - Alto custo para } \\
\text { implantação do modelo. } \\
\text { - Retornos não imediatos. }\end{array}$ \\
\hline
\end{tabular}

Quadro 2 - Análise de SWOT do modelo elaborado por Campos e Selig (2002).

O modelo de Campos e Selig (2002) apresenta forças relacionadas às teorias usadas, a possibilidade de estruturar o modelo para diferentes atividades e por apresentar resultados da aplicação do modelo.

Este modelo faz o uso de teorias ligadas ao Balanced Scorecard e a gestão ambiental, através da integração das fases de implantação da gestão ambiental apresentadas na ISO 14001 junto as fases de implantação de um BSC. Com isso, não favorece a nenhuma atividade específica, podendo ser estruturado para qualquer atividade. Além disso, os autores apresentam resultados de uma aplicação do modelo, o que possibilita uma melhor avaliação da aplicabilidade do modelo.

As fraquezas apresentadas no modelo estão relacionadas à forma como o modelo é estruturado, pois apresenta várias etapas, subdividida em etapas internas, o que não facilita a sua implantação, como também, demanda muito capital humano. Outra fraqueza do modelo está relacionada à ênfase atribuída na parte operacional, uma vez que a maior parte das etapas faz referência à operacionalidade.

Dessa forma, a estrutura do modelo proposto favorece mais os aspectos operacionais. A estrutura do modelo possibilita a incorporação dos aspectos sociais, por permitir adaptações. Com esta inclusão, o modelo pode direcionar a empresa a uma sustentabilidade empresarial, atendendo o mercado contemporâneo de empresas que procuram por uma sustentabilidade empresarial.

Outra oportunidade do modelo proposto é possibilidade a da empresa que implantar o modelo de obter certificações ambientais, devido ao fato de implantar 
um sistema de gestão ambiental. E com isso, a empresa poderá competir em mercados externos, uma vez que muitos mercados já se exigem certificações ambientais.

Entretanto o modelo não apresenta resultados imediatos, pois é preciso realiza avaliações antes da implementação do modelo. Além disso, o modelo exige dispêndios financeiros para a implantação.

O segundo modelo analisado foi o modelo elaborado por Yongvanich e Guthrie (2006). Os resultados estão apresentados no quadro 3.

\begin{tabular}{|c|c|c|}
\hline & ositivo & $\mathrm{Neg}$ \\
\hline 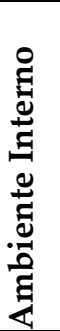 & $\begin{array}{l}\text { FORÇAS } \\
\text { - Teorias usadas favorecem a } \\
\text { sustentabilidade empresarial. } \\
\text { - Favorecimento dos aspectos } \\
\text { administrativos. }\end{array}$ & $\begin{array}{l}\text { FRAQUEZAS } \\
\text { - Os objetivos das categorias } \\
\text { do modelo não são } \\
\text { apresentados. } \\
\text {-Falta de mensuração do } \\
\text { modelo. }\end{array}$ \\
\hline 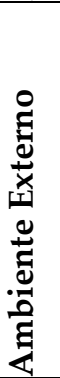 & $\begin{array}{l}\text { OPORTUNIDADES } \\
\text { - Expansão do número de } \\
\text { empresas voltadas para a } \\
\text { sustentabilidade empresarial. } \\
\text { - Possibilidade de atender as } \\
\text { necessidades de empresas que } \\
\text { buscam a sustentabilidade. }\end{array}$ & $\begin{array}{l}\text { AMEAÇAS } \\
\text { - Difícil implantação dos } \\
\text { modelo } \\
\text { - Necessidade de grandes } \\
\text { mudanças na empresa. } \\
\text { - Retornos não imediatos. }\end{array}$ \\
\hline
\end{tabular}

Quadro 3 - Análise de SWOT do modelo elaborado por Yongvanich e Guthrie (2006).

O modelo considera não só os aspectos financeiros e ambientais, como também os aspectos sociais. Isto ocorre, devido a integração do BSC, capital humano e relatórios sociais e ambientais.

O uso destas diferentes teorias favorece para a adoção da sustentabilidade empresarial, pois valoriza os aspectos sociais, ambientais e financeiros. Além disto, valorizar os aspectos administrativos, através da elaboração de relatórios sociais e ambientais, voltados para todos os usuários que necessitam das informações.

No entanto, ele não apresenta os objetivos das categorias relacionadas ao capital humano, capital externo e estrutura interna, o que pode acarretar em uma implantação inadequada do modelo. No modelo são apresentados apenas os possíveis indicadores a serem usados para a avaliação das categorias. Outra fraqueza do modelo é a falta de apresentação de resultados aplicados. A apresentação do modelo é dada apenas de forma teórica, o que tende a não facilita aplicabilidade do modelo. 
O modelo possibilita atender as necessidades das empresas que buscam a sustentabilidade empresarial, pois a sua estrutura favorece. Outro aspecto importante, neste contexto, e a expansão dos mercados e das empresas que procuram uma sustentabilidade dos seus produtos e das suas atividades. Com isto, o modelo pode favorecer estes mercados e empresas com pretensões sustentáveis.

Contudo, o modelo tem uma proposta de difícil estruturação, pois são apresentadas as categorias com os possíveis indicadores, entretanto não são apresentados os objetivos destes indicadores, são exigidas, também, modificações internas para estruturar as categorias do modelo, o que dificulta a implantação do modelo. Além disso, ele não apresenta a possibilidade de obtenção de resultados imediatos.

O terceiro modelo analisado foi o modelo elaborado por Bonacchi e Rinaldi (2007). Os resultados estão apresentados no quadro 4.

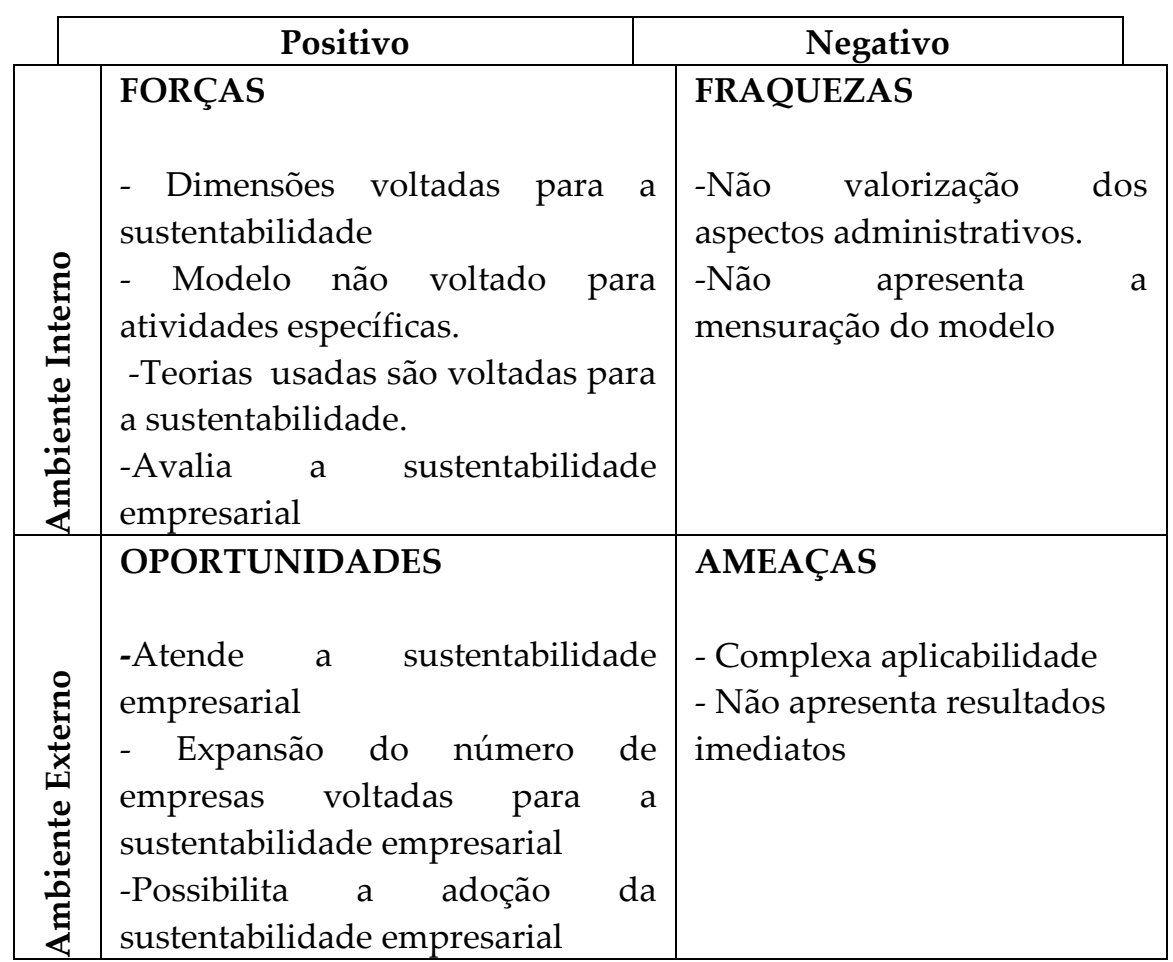

Quadro 4 - Análise de SWOT do modelo elaborado por Bonacchi e Rinaldi (2007).

O modelo de é estruturado com base no BSC, propondo dimensões voltadas para a obtenção da sustentabilidade empresarial, o que segundo os autores facilita a adoção da sustentabilidade por parte da empresa.

As teorias usadas no modelo favorecem a sua adoção, porque usa não só BSC para estruturar o modelo, como também faz uso da sustentabilidade. Dessa forma, ele não é voltado para nenhuma atividade específica e a sua estrutura possibilita que a sustentabilidade seja mensurada. 
No entanto, as dimensões da sustentabilidade propostas no modelo, em nenhum momento valorizam os aspectos administrativos, uma vez que as dimensões propostas são voltadas para a parte operacional. $\mathrm{O}$ modelo também não apresenta resultados aplicados, a apresentação da aplicabilidade é dada apenas teoricamente.

O modelo possibilita a adoção da sustentabilidade na empresa, permitindo a mensuração da sustentabilidade empresarial, através de indicadores. Com isso, o modelo pode atender as empresas que buscam a implementação e a avaliação da sustentabilidade.

Entretanto, a aplicação do modelo não pode ser dada de forma imediata, o modelo apresenta uma estrutura complexa, que para a sua implantação faz-se necessário que sejam avaliados os objetivos da empresa, para evitar divergências com a proposta da sustentabilidade. Dessa forma, os resultados com a implantação do modelo não são imediatos.

\section{CONSIDERAÇÕES FINAIS}

Com base no levantamento da análise de SWOT dos modelos e com base na literatura existente sobre Balanced Scorecard sustentável foram caracterizadas e analisadas as características dos modelos de Balanced Scorecard estruturados sob a ótica da sustentabilidade empresarial para empresas que atuam no agronegócio, no sentido de responder ao objetivo geral da pesquisa.

Assim, os modelos de Balanced Scorecard sustentável voltados para empresas que atuam no agronegócio, devem apresentar nas suas estruturas dimensões que valorizem não só o processo operacional, como também a parte administrativa, e com isso, poderia usar na estruturação do Balanced Sorecard Sustentável dimensões relacionadas às questões: ambientais, financeiras, sociais, operacionais e administrativas.

Para isso, o modelo pode fazer o uso da ISO 14001, Balanced Scorecard, sustentabilidade. O uso da sustentabilidade seria para parte das dimensões, o uso da ISO 14001 seria para estruturar as perspectivas operacionais e administrativas, assim como sugerir indicadores, e o uso do Balanced Scorecard seria para a estruturação dos modelos, através do levantamento da visão, missão, interligando as perspectivas, de forma a integrar as estratégias sustentáveis às estratégias da empresa.

Dessa forma, a operacionalização dos modelos deverá ser realizada com base na integração direta e indireta das dimensões, ou seja, devem ser traçadas metas principais de cada uma das dimensões fazendo uma interligação direta e indireta destas metas entre as dimensões traçadas. Assim, todos os objetivos e as metas destas dimensões deverão atender a sustentabilidade, e deverá ser feito o uso de 
SILVA, M. D. de O. P.; CALLADO, A. A. C. Análise de Modelos de Balanced...

indicadores para avaliar o desempenho das dimensões de cada uma das dimensões, assim como para medir a sustentabilidade da empresa.

Os objetivos dos modelos voltados para empresas que atuam no agronegócio deveram ter o sentido de integrar medidas financeiras e não financeiras derivadas das estratégias, de forma a equilibrar as medidas para a obtenção da sustentabilidade empresarial. Contudo, a pesquisa limitou-se a levantar de forma descritiva características dos modelos de Balanced Scorecard sustentável.

A partir das evidências do presente trabalho, sugere-se a título de pesquisas futuras que possam existir aprofundamentos deste estudo, reaplicando-o nas demais possibilidades de elaboração de modelos de Balanced Scorecard, através de novas análises sob diferentes perspectivas, assim como, propostas de elaboração de novos modelos de Balanced Scorecard sustentável. Visto que, o futuro dos negócios empresariais dependerá da sustentabilidade empresarial.

\section{REFERÊNCIAS}

ANDRADE, M. M. de. Introdução à metodologia do trabalho científico. São Paulo: Atlas; 1999.

AZZONE, G. et al. Defining Environmental Performance Indicators: an integrated Framework. Business Strategy and the Environment, [S.1], v. 5, n. 2, p. 69-80, 2006.

BARBOSA JUNIOR, A. S.; et al. Balanced Scorecard: uma análise dos impactos de sua implantação em indicadores financeiros de empresas brasileiras. In: CONGRESSO BRASILEIRO DE CUSTOS, 13., 2006. Belo Horizonte. Anais... Belo Horizonte: CBC, 2006. CD-ROM.

BANEGAS, O; R.; NEVADA, P. D; TEJADA, P. Á. El cuadro de mando como instrumento de control en la gestión social: recursos humanos y medio ambiente. Revista Española de Financiación y Contabilidad, [S.1], n. 103, p. 107-147, ene./mar. 2000.

BEJA, R. Balanced Scorecard e Desenvolvimento Sustentável. Revisores e Empresas, [S.1], n. 23, p. 7-11, out./dez. 2003

BIEKER, T. Managing Corporate Sustainability with the Balanced Scorecard: developing a Balanced Scorecard for Integrity Management. St. Gallen: University of St. Gallen ,2002. 
SILVA, M. D. de O. P.; CALlADO, A. A. C. Análise de Modelos de Balanced...

BIEKER, Thomas. Sustainability management with the Balanced Scorecard. [S.1 : S.n], 2005. International Summer Academy on Technology Studies - Corporate Sustainability.

BIEKER, T. e GMINDER, C. Towards a Sustainability Balanced Scorecard. [S.1 : S.n], 2001. Oikos PhD Summer Academy, Foundation for Economy and Ecology, University of St.

Gallen.

BIEKER, T. e GMINDER, C. Managing Corporate Social Responsibility by using the "Sustainability-Balanced Scorecard". [S.1 : S.n], 2002. International Conference of the Greening of Industry Network, June 2002, Göteborg (Sweden).

BIEKER, T; WAXENBERGER, B. Sustainability Balanced Scorecard Business Ethics Developing a Balanced Scorecard for Integrity Management, 2002, Sweden. In International Conference of the Greening of Industry Network, 10. Göteborg/Sweden.

BIEKER, T. et al. Towards a Sustainability Balanced Scorecard Linking Environmental and Social Sustainability to Business Strategy. 2001. Disponível em: $<$ http://jobfunctions.bnet.com/abstract.aspx?docid=138479> Acesso em: jun. 2010.

BONACCHI, M; RINALDI, L. Sustainable Development Performance And Sustainability: are Stakeholders The Missing Link? Invited Seminars of the University of Virginia, Darden School of Business (USA), September 24, 2007.

CARVALHO, G. da S. C.; TOSTES, F. P. A implantação do Balanced Scorecard em uma empresa brasileira do setor petroquímico: um estudo de caso. 2007. In: CONGRESSO BRASILEIRO DE CUSTOS, 14., 2007, João Pessoa. Anais...João Pessoa: [S.n], 2007

COSTA, A. P. P. Contabilidade Gerencial: um estudo sobre a contribuição do Balanced Scorecard. São Paulo: FEA/USP, 2001.

CAMPOS, L.; SELIG, P. SGADA. Sistema de Gestão e Avaliação do desempenho ambiental: a aplicação de um modelo de SGA que utiliza o Balanced Scorecard (BSC). Revista Read, v. 8, n. 6, p. 1-23, nov./dez, [2002] 
SILVA, M. D. de O. P.; CALlADO, A. A. C. Análise de Modelos de Balanced...

DURÁN, O; PUGLIA, V. B. SCORECARD AMBIENTAL: monitoração dos custos ambientais através da web. Revista chilena de ingeniería, v. 15, n. 3, p. 291-301, 2007.

FIGGE, F.; HAHN, T.; SCHALTEGGER, S.; WAGNER, M. The Sustainability Balanced Scorecard - Linking Sustainability Management to Business Strategy. 2002. Business Strategy and the Environment, September/October, 11(5). 269-284.

GARCÍA, J. G. Perspectiva ambiental del cuadro de mando integral de una organizacion. Aplicacion de la inteligencia de negocio. Ingenieria Quimica - Madrid, v. 39, n. 448, p. 70-79, 2007.

GIL, A. C. Métodos e Técnicas de Pesquisa Social. 5. ed. São Paulo: Atlas, 1999.

GIOLLO, P. R. Modelo de Avaliação de Desempenho fundamentado no Balanced Scorecard: um estudo de caso da URI - Campus Erechim. Erechim: UFRGS, 2002. Dissertação.

GIUNTINI, N. Contribuição ao estudo de utilidade do Balanced Scorecard: um estudo de caso de uma empresa siderúrgica e metalúrgica operando no Brasil. Dissertação. Mestrado em Controladoria e Contabilidade Estratégica. Centro Universitário Alvares Penteado - UNIFECAP, 2003.

HAMMER, B. Integrating Market-Based Sustainability Indicators and Performance Management Systems. Managing For Sustainability Using Performance Systems. [S.1 :S.n], 2005.

HERRERO, E. Balanced Scorecard e a gestão estratégica: uma abordagem prática. 5 reimpressão. Rio de Janeiro: Elsevier, 2005.

HOCKERTS, K; O'ROURK, A. The Balanced Scorecard a tool to manage social sustainability? In: International conference of the Greening of Industry Network, 2002. 10., Sweden. June 23-26, Göteborg, Sweden.

HOCKERTS, K. Corporate Sustainability Management - Towards controlling corporate ecological and social sustainability. Sustainability at the Millenium: Globalization, Competitiveness and the Public Trust. January 21-25, 2001. Ninth International Conference of Greening of Industry Network Bangkok. 
SILVA, M. D. de O. P.; CALlADO, A. A. C. Análise de Modelos de Balanced...

HUBBARD, G. Measuring Organizational Performance: Beyond the Triple Bottom Line. 2009. Business Strategy and the Environment, March, 18(3). 177-191.

JOHNSON, S. Identification and Selection of Environmental Performance Indicators: Application of the Balanced Scorecard Approach. 1998. Corporate Environmental Strategy, Summer, 5(4). 34-41.

KAPLAN, R.; NORTON, D. A estratégia em ação: Balanced Scorecard. Rio de Janeiro: Elsevier, 1997.

LÄNSILUOTO, A.; JÄRVENPÄ $\ddot{A}$, M. Sustainability and Performance Management - A Case of Implementation of Sustainable Balanced Scorecard in a Food Processing Company. 2007. Comunicação apresentada no 30th Annual Congress of the European Accounting Association, 25-27 April, Lisboa.

MACEDO, A. V. P.; QUEIROZ, M. E. A. Gerenciando e Otimizando a Sustentabilidade Empresarial através da Ferramenta Balanced Scorecard: em busca da mensuração. In: Congresso Virtual Brasileiro de Administração, 2007.

MARIAN, S. et al. O processo de implantação do Balanced Scorecard na Logística estudo de caso no Porto de Itajai. In: Congresso Brasileiro de Custos, 15., 2008. Curitiba. Anais... Curitiba: CBC, 2008. CD-ROM.

MARTINSONS, M.; DAVISON, R.; TSE, D. The balanced scorecard: a foundation for the strategic management of information systems. Decision Support Systems, 1999, v. 25, p. 71-88, 1999.

MÖLLER, A; SCHALTEGGER, S.The Sustainability Balanced Scorecard as a Framework for Eco-Efficiency Analysis. 2005. Journal of Industrial Ecology, Fall, 9(4). 73-83.

MONTEIRO, P.; CASTRO, A.; PROCHNIK, V. A mensuração do desempenho ambiental no Balanced Scorecard e o Caso da Shell. 2003. Comunicação apresentada no VII Encontro Nacional sobre Gestão Empresarial e Meio Ambiente, Outubro, Brasil.

QUESADO, P. R.; RODRIGUES, L. L. O Balanced Scorecard e a gestão ambiental. 2009. III Congrés Català de Comptabilitat i Direcció. ESADE. Barcelona. 
SILVA, M. D. de O. P.; CALlADO, A. A. C. Análise de Modelos de Balanced...

ROCHA, J.; NEVES, R.; SELIG, P. Balanced Scorecard na Gestão Ambiental. . In: Congresso Interamericano sobre o Meio Ambiente, 7., 2001, Chile, Anais...Chile, 2001, p. 5-7.

SIDIROPOULOS, M.; MOUZAKITIS, Y.; ADAMIDES, E.; GOUTSOS, S. Applying Sustainable Indicators to Corporate Strategy:The Eco-balanced Scorecard. Environmental research, engineering and management, 2004. n.1(27), p. 28-33. 2004.

SILVA, J. G. S. da. Plano de Implantação do Balanced Scorecard em uma empresa industrial do Ramo Couro Calçadista. 2006. Dissertação. Universidade Federal do Rio Grande do Sul. Disponível em: <http://www.lume.ufrgs.br/handle/10183/12583> Acesso em: fev. 2010.

SOARES, C. R. D. Desenvolvimento de uma sistemática de elaboração do Balanced Scorecard para pequenas empresas. 2001. Dissertação. Disponível em : <http://www.lume.ufrgs.br/handle/10183/1517?show=full> Acesso: fev. 2010.

VERGARA, S. C. Projetos e relatórios de pesquisa em administração. 10. ed. São Paulo: Atlas, 2009.

YONGVANICH, K.; GUTHRIE, J. An Extended Performance Reporting Framework for Social and Environmental Accounting. 2006. Business Strategy and the Environmental, Sep.-oct., 15(5). 309-321.

ZINGALES, F.; HOCKERTS, K. Balanced Scorecard and Sustainability: Examples from Literature and Practice. 2003. Centre for the Management of Environmental Resources, Working Papers Series, 2003/30/CMER. 